\title{
The apoptosis associated tyrosine kinase gene is frequently hypermethylated in human cancer and is regulated by epigenetic mechanisms
}

\author{
Tanja Haag ${ }^{1}$, Christina E. Herkt ${ }^{1}$, Sara K. Walesch ${ }^{1}$, Antje M. Richter ${ }^{1}$ and Reinhard \\ H. Dammann ${ }^{1}$ \\ ${ }^{1}$ Institute for Genetics; Justus-Liebig-University; Universities of Giessen and Marburg Lung Center, Member of the German \\ Center for Lung Research; Giessen, Germany \\ Correspondence to: Reinhard H. Dammann, email: Reinhard.Dammann@gen.bio.uni-giessen.de \\ Keywords: AATK/ epigenetic regulation / DNA methylation/ human cancer/ tumor suppressor/ CTCF \\ Received: July 30, $2014 \quad$ Accepted: August 18, $2014 \quad$ Published: August 19, 2014
}

This is an open-access article distributed under the terms of the Creative Commons Attribution License, which permits unrestricted use, distribution, and reproduction in any medium, provided the original author and source are credited.

\section{ABSTRACT:}

Epigenetic gene inactivation through promoter hypermethylation is an important aberration involved in the silencing of tumor-associated genes in cancer. Here we identified the apoptosis associated tyrosine kinase (AATK) as an epigenetically downregulated tumor related gene. We analyzed the epigenetic regulation of AATK in several human cancer cell lines and normal tissues by methylation and expression analysis. Hypermethylation of AATK was also analyzed in 25 primary lung tumors, 30 breast cancers and 24 matching breast tissues. In normal tissues the AATK CpG island promoter was unmethylated and AATK was expressed. Hypermethylation of AATK occurred frequently in 13 out of 14 (93\%) human cancer cell lines. Methylation was reversed by 5-aza-2'-deoxycytidine treatment leading to re-expression of AATK in cancer cell lines. Aberrant methylation of AATK was also revealed in primary lung $(40 \%)$ and breast $(53 \%)$ cancers, but was found to be significantly less methylated in matching normal breast tissues $(17 \% ; p<0.01)$. In addition, we observed that $A A T K$ is epigenetically reactivated through the chromatin regulator CTCF. We further show that overexpression of Aatk significantly suppresses colony formation in cancer cell lines. Our findings suggest that the apoptosis associated tyrosine kinase is frequently inactivated in human cancers and acts as a tumor suppressive gene.

\section{INTRODUCTION}

Epigenetic modifications are important regulatory mechanisms for initiating and maintaining memory effects on gene expression. In mammals, these epigenetic mechanisms play essential roles in normal development through their effect on gene imprinting, X-chromosome inactivation and transcriptional inactivation of repetitive genomic elements. Moreover, epigenetic silencing of tumor suppressor genes is frequently observed in cancer [1]. In particular, de novo methylation of $\mathrm{CpG}$ island promoters is a hallmark of gene silencing during malignant transformation. $\mathrm{CpG}$ islands are sequences greater than $500 \mathrm{bp}$ of $\mathrm{GC}$-rich and $\mathrm{CpG}$-dense elements in the genome. About $70 \%$ of known genes harbor a $\mathrm{CpG}$ island within $-1 \mathrm{~kb}$ to $+1 \mathrm{~kb}$ of their transcription initiation site. During tumorigenesis $\mathrm{CpG}$ island promoters become hypermethylated and this alteration is accompanied by the formation of a repressive chromatin and transcriptional silencing. Tumor suppressor genes that are frequently epigenetically inactivated are the Ras association domain family $1 A$ (RASSF1A) gene and the cyclin-dependent kinase inhibitor $2 A$ (p16) [1-3].

The CCCTC binding factor (CTCF) is a zinc fingerencoding protein involved in imprinting and chromosomal gene organization [4]. Several findings suggest that the CTCF insulator protein may contribute the boundaries at $\mathrm{CpG}$ island promoters [5-8]. Binding of $\mathrm{CTCF}$ is maintained in mitotic chromatin and may provide an epigenetic memory during cell division of proliferating cells [9]. Disruption of molecular boundaries mediated by CTCF may facilitate the epigenetic silencing of tumor suppressor genes $[10,11]$. Recently, it has been shown that epigenetic downregulation of p16 and RASSF1A is 
associated with loss of CTCF binding and disappearance of a chromatin boundary [12].

The apoptosis associated tyrosine kinase (AATK) gene is localized on chromosome 17 at q25.3 [13]. Deletion of $17 \mathrm{q} 25.3$ was reported for several human cancers including oral, cervical and breast cancer [1416]. The AATK protein that is also named AATYK or LMTK1 (lemur tyrosine kinase 1) consists of 1374 aa with the $\mathrm{N}$-terminus harboring a protein tyrosine kinase domain (Fig. 1) [17]. AATK promotes neuronal differentiation and is induced during growth arrest and apoptosis of myeloid cells [18, 19]. Downregulation of AATK expression was reported for adenocarcinoma of the colon and for melanomas [20,21]. In melanoma cells AATK overexpression inhibits growth and migration, and promotes apoptosis [21]. In our study we report frequent epigenetic inactivation of $A A T K$ in different human cancer entities (e.g. breast and lung) and its growth suppressive function in lung cancer. Furthermore, we show that the chromatin regulator $\mathrm{CTCF}$ induces epigenetic reactivation of $A A T K$.

\section{RESULTS}

\section{Methylation of AATK occurs in human cancers}

We have performed a genome wide methylation screen in the lung cancer cell line H322 and have revealed a promoter specific hypermethylation of the apoptosis associated tyrosine kinase (AATK) gene (data not shown). The schematic promoter region of $A A T K$ and corresponding $\mathrm{CpG}$ islands is shown in Fig. $1 \mathrm{~A}$. The promoter lies within a $\mathrm{CpG}$ island of $527 \mathrm{bp}$ on chromosome 17 from position 79'139'502 to 79'140'028 (UCSC genome browser).

A

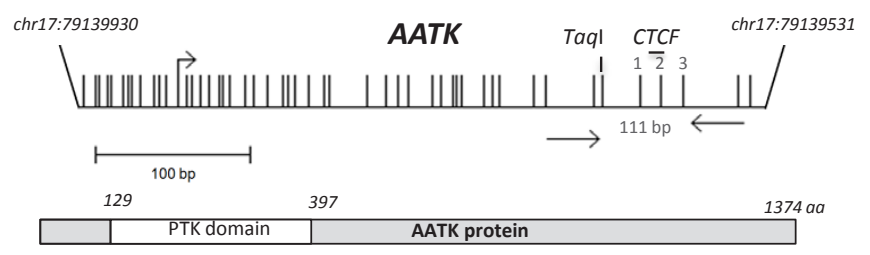

B

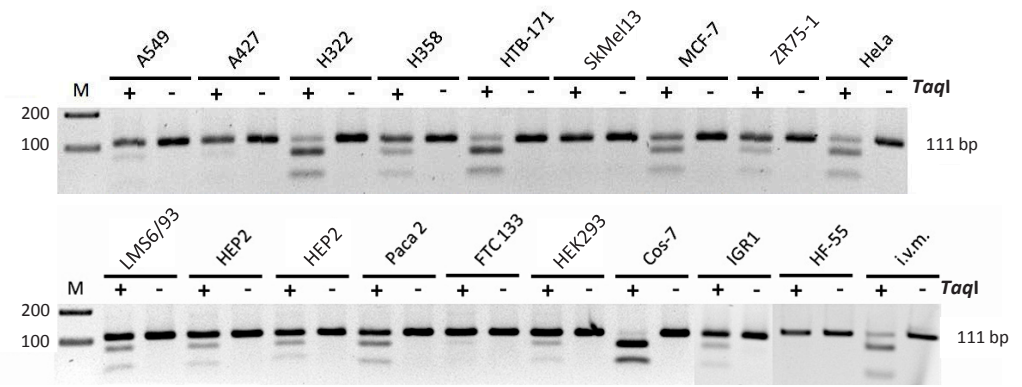

C

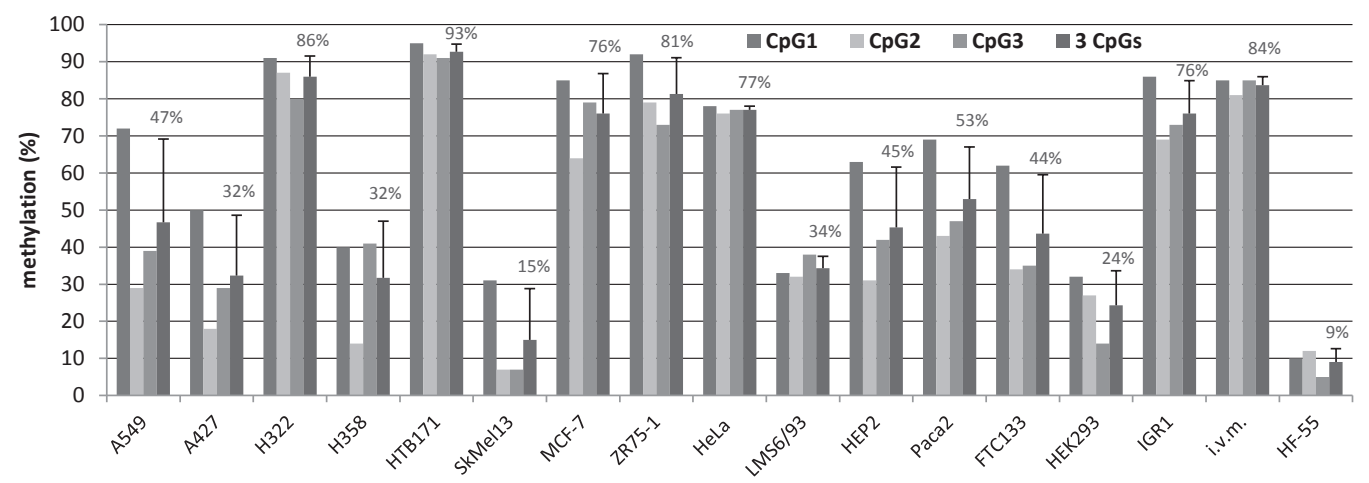

Fig. 1: Hypermethylation of AATK in human cancers. A. Structure of the AATK CpG island promoter on chromosome 17 and the AATK protein. Arrows mark transcriptional (+1) start site for AATK. Vertical lines indicate CpGs. The 111 bp PCR product with respective primers and the TaqI site are depicted. The CTCF binding site is shown. The protein tyrosine kinase (PTK domain) of AATK is marked. B. Combined bisulfite restriction analysis of AATK. Bisulfite-treated DNA from the indicated cancer cell lines, human fibroblasts (HF-55) and in vitro methylated DNA (i.v.m.) was amplified, digested with TaqI (+) or mock digested (-) and resolved on $2 \%$ gels with a 100 bp marker (M). C. Bisulfite pyrosequence analysis of AATK. The methylation levels of three CpGs of the PCR products were analyzed by pyrosequencing. 
A

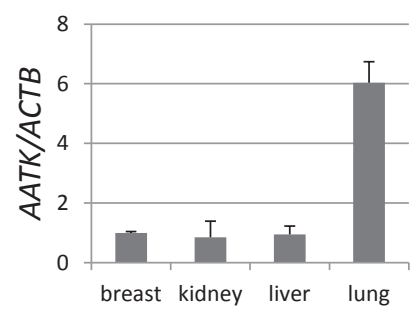

B

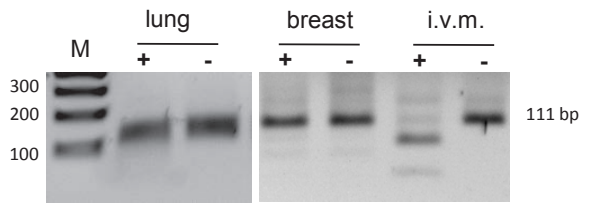

$\mathrm{C}$
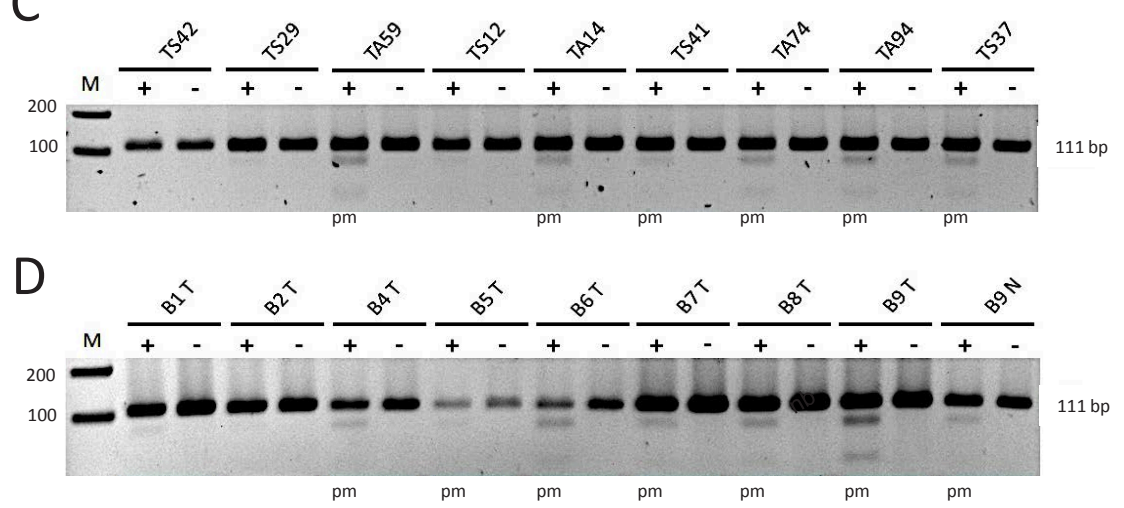

Fig. 2: Expression and methylation of AATK in normal tissues and primary tumors. A. Expression of $A A T K$ was analyzed in normal breast, kidney, liver and lung tissues by quantitative RT-PCR and normalized to $A C T B$ levels (breast=1). B. Methylation of $A A T K$ was analyzed in normal lung and breast tissues and in vitro methylated DNA by COBRA. Mock digest (-) and TaqI digest (+) are shown. C. Methylation analysis is shown for primary lung cancer samples $(\mathrm{TA}=$ adenocarcinoma, $\mathrm{TS}=$ squamous cell carcinoma). $\mathrm{D}$. Methylation analysis in primary breast cancer ( $\mathrm{T}=$ tumor, $\mathrm{N}=$ corresponding normal tissue). Products were resolved on a $2 \%$ gel with a $100 \mathrm{bp}$ marker (M). (pm=partially methylated).

A

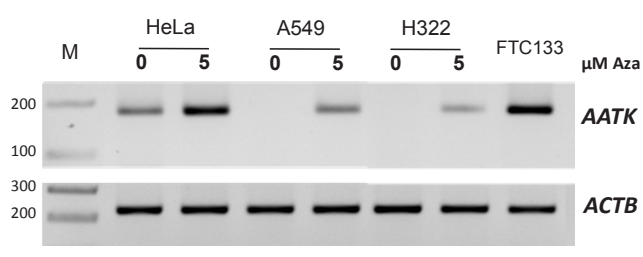

C

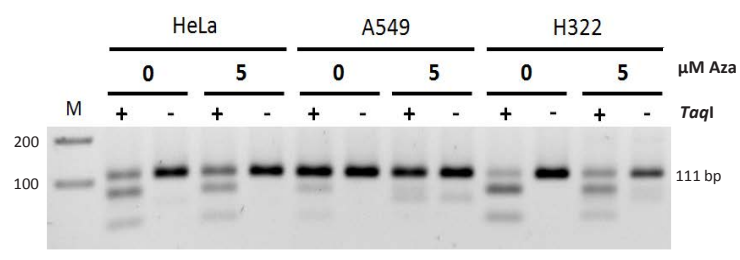

B

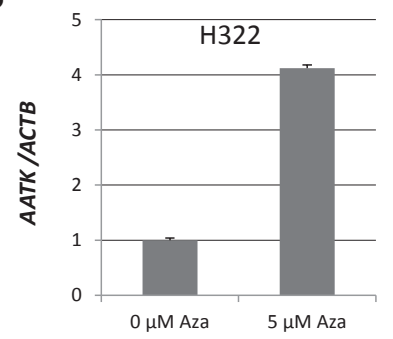

D

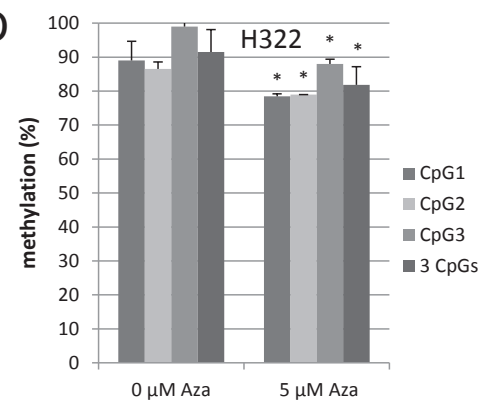

Fig. 3: AATK expression in cancer cells, reexpression and demethylation under Aza treatment. A. Expression analysis of $A A T K$ is shown after Aza treatment $(0$ and $5 \mu \mathrm{M})$ in HeLa, lung cancer cell lines A549 and H322 and thyroid cancer cell line FTC133. AATK (170 bp) and ACTB (226 bp) levels were analyzed by RT-PCR after four days of Aza treatment on a $2 \%$ gel. B. Expression of AATK was analyzed in $\mathrm{H} 322$ cells by quantitative RT-PCR after Aza treatment and normalized to ACTB. C. COBRA analysis is shown after Aza treatment. Product of TaqI digest (+) and mock digest (-) were resolved on a $2 \%$ gel with a 100 bp marker (M). D. AATK methylation analysis was performed in lung cancer $\mathrm{H} 322$ and quantified by bisulfite pyrosequencing. Three $\mathrm{CpGs}$ are included in analyzed region and according mean and SD are shown. P values were calculated using two tailed $t$-test $(*=p<0.05)$. 
To reveal the epigenetic status of $A A T K$ in human cancers in more detail, we have analyzed the aberrant methylation of AATK in lung cancer (A549, A427, H322, H358, HTB-171), breast cancer (MCF-7, ZR751), melanoma (Sk-Mel13, IGR1), leiomyosarcoma (LMS6/93), follicular thyroid (FTC133), larynx cancer (HEP2), pancreas carcinoma cell line (PaCa2), cervix cancer (HeLa), HEK293 and human fibroblast (HF-55) by COBRA (Fig. 1B). Fragmentation of the PCR product indicates an underlying methylated $A A T K$, whereas undigested PCR products originate from unmethylated AATK $\mathrm{CpG}$ island. In vitro methylated genomic DNA (i.v.m.) served as a methylated control (Fig. 1B). Normal human fibroblast (HF-55) and melanoma cells (Sk-Mel13) were unmethylated as analyzed by COBRA. COBRA data were confirmed by genomic bisulfite pyrosequencing of three CpGs within the AATK CpG island promoter (Fig. $1 \mathrm{~A}$ and C). All lung cancer cell lines (A549, A427, H322, H358 and HTB171) were partially methylated for AATK (Fig. 1B and C). For breast cancer cell lines (MCF-7, ZR75-1), HeLa, LMS6/93, HEP2, Paca2, FTC133 and IGR1 partial methylation of $A A T K$ was also observed (Fig. 1B and C). Thus, a total of 15 cancer cell lines were analyzed, of which 14 (93\%) were methylated for AATK. Therefore frequent hypermethylation of $A A T K$ was found in different human cancer entities, including lung and breast cancers.

\section{AATK hypermethylation in primary human breast and lung cancers}

To analyze the impact of epigenetic regulation of $A A T K$ in carcinogenesis we investigated its expression and methylation in normal tissues as well as in breast and lung cancer samples (Fig. 2). Expression of AATK was found in normal breast, kidney and liver tissues and the highest expression was observed in normal lung tissues (Fig. 2A). AATK was unmethylated in normal lung and in three breast tissues isolated from healthy patients (Fig. 2B and data not show). Next we analyzed the aberrant methylation of $A A T K$ in 25 primary lung and 30 breast cancers by COBRA (Fig. 2). Four out of five lung adenocarcinomas (e.g. TA59) exhibited a partial methylation of AATK (Fig. 2C). However, aberrant AATK methylation was found in only two out of eight squamous lung tumors (e.g. TS37; Fig. 2C). In small cell lung cancer $A A T K$ was hypermethylated in four out of 12 tissues (data not shown). Thus, aberrant methylation of $A A T K$ was observed in 10 out of $25(40 \%)$ of lung cancer samples. We also analyzed 30 primary breast tumors and 24 corresponding matching tissue controls (Figure 2D). 16 out of 30 (53\%) breast cancer tissues were methylated, but $A A T K$ methylation was only found in 4 out of $24(17 \%)$ matching control tissues $(p<0.01$, two tailed Fisher's exact test). Moreover, methylation was weaker in the normal samples than in the corresponding tumor tissue (e.g. B9N and B9T, respectively; Fig. 2D). In summary, hypermethylation of $A A T K$ was also demonstrated in primary tumor tissues of cancer patients.

\section{Decreased expression of AATK is associated with its hypermethylation in human cancer cell lines}

5-Aza-2'-deoxycytidine (Aza) inhibits DNA methylation [22] and is known to reverse hypermethylation of tumor suppressor genes, which can further lead to their re-expression [2]. We therefore chose HeLa, A549 and $\mathrm{H} 322$ with a methylated $A A T K \mathrm{CpG}$ island for Aza treatment and analyzed $A A T K$ expression by RT-PCR as well as its methylation status (Fig. 3). The lung cancer cell line $\mathrm{H} 322$ has a methylated $A A T K$ promoter (Fig. 1 and 3) and shows very low endogenous $A A T K$ expression (Fig. 3A and B). RT-PCR shows that $5 \mu \mathrm{M}$ Aza leads to $A A T K$ re-expression (Fig. $3 \mathrm{~A}$ and $\mathrm{B}$ ). Similar results are observed for the lung cancer cell line A459 and for HeLa cells, which are partially and strongly methylated respectively (Fig. 1 and 3). In HeLa and A549 the AATK expression is increased upon Aza treatment (Fig. 3A). FTC133 is partially methylated for the AATK promoter (Fig. 1B) and $A A T K$ expression is comparable to the Azatreated HeLa cells (Fig. 3A). The re-expression under Aza
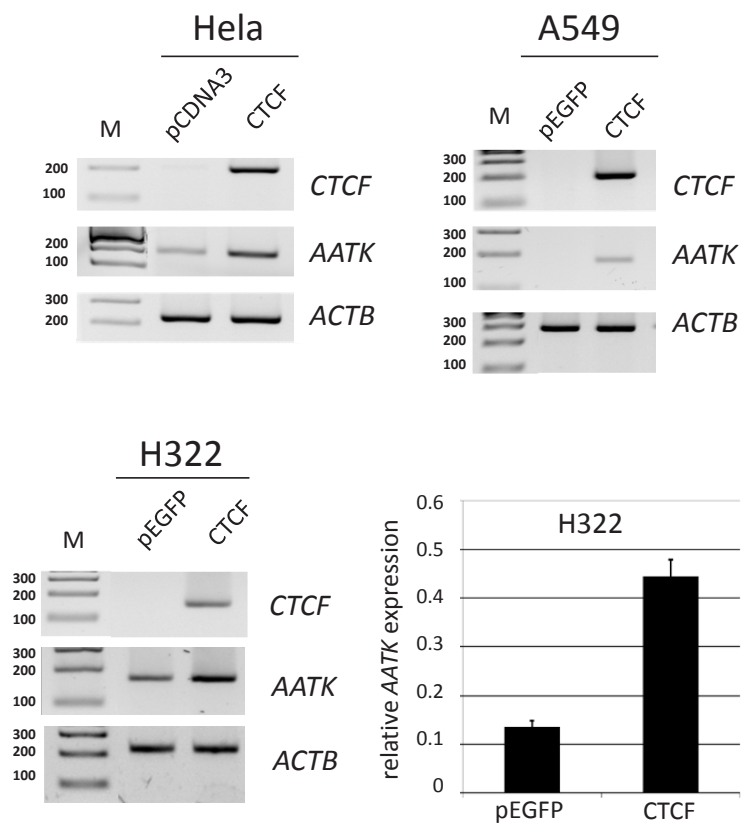

Fig. 4: CTCF induced reexpression of AATK. Expression analysis of HeLa, lung cancer cells A549 and H322 after CTCF and control transfection. CTCF was cloned in the corresponding vector (pCDNA3 or pEGFP) and transfected. After one to two days RNA was isolated and expression of AATK (170 bp), CTCF (199 bp) and ACTB (226 bp) was analyzed by RT-PCR on a $2 \%$ gel with a 100 bp ladder (M). For H322 AATK levels were analyzed by qRT-PCR and normalized and plotted relative to $A C T B(=1)$. 
treatment for HeLa, A549 and H322 was accompanied by AATK demethylation (Fig. 3C and D). H322 were 92\% methylated and under Aza treatment methylation decreased significantly to $82 \%(5 \mu \mathrm{M}$ Aza) $(\mathrm{p}=0.01$, t-test; Fig. 3D). Genomic bisulfite pyrosequencing revealed a significant $10 \%$ demethylation at all three analyzed CpGs ( $<<0.05$; Fig. 3D). In cancer cells, we observed an epigenetic silencing of the $A A T K \mathrm{CpG}$ island promoter, which was reversed by Aza treatment as shown by AATK re-expression and promoter demethylation.

\section{Regulation of AATK by the insulator protein CTCF}

Previously, it has been reported that the CTCCC binding protein (CTCF) is involved in the epigenetic regulation of tumor suppressor genes [12]. Wendt and Barksi et al. have reported that downregulation of CTCF by RNA interference caused a twofold repression of $A A T K$ in HeLa cells $[23,24]$. Database analysis of CTCF

A

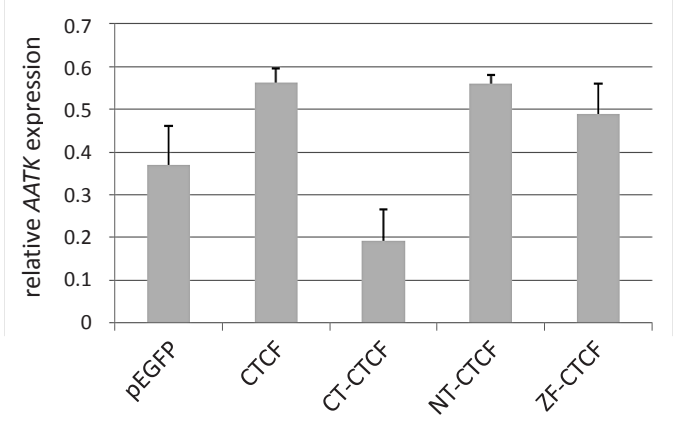

B

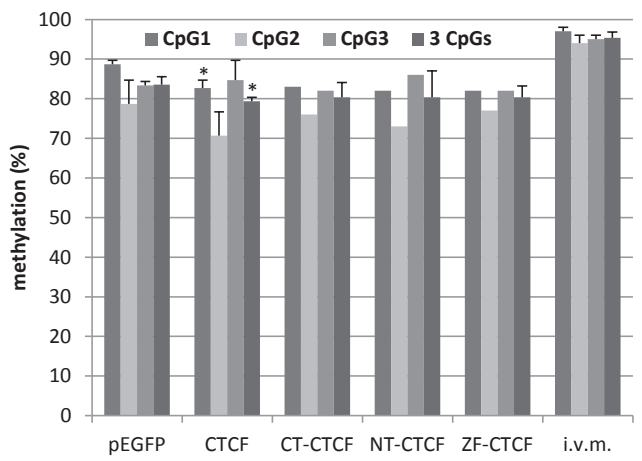

Fig. 5: CTCF induced reexpression and demethylation of AATK. A. Expression analysis of AATK after CTCF and control transfection. Different constructs of chicken $\mathrm{CTCF}$ ( $\mathrm{CT}=\mathrm{C}$-terminal domain, $\mathrm{NT}=\mathrm{N}$-terminal domain and $\mathrm{ZF}=$ zinc finger domain) were generated and transfected in H322 lung cancer cells. After two days RNA was isolated and AATK expression was analyzed by qRT-PCR and then normalized and plotted relative to $A C T B(=1) \mathrm{B}$. CTCF induced demethylation of AATK. Methylation analysis was performed in $\mathrm{H} 322$ after CTCF and control (pEGFP) transfection. The average methylation of three $\mathrm{CpGs}$ was quantified by bisulfite pyrosequencing. $\mathrm{P}$ values were calculated using two tailed t-test. (i.v.m. = in vitro methylated DNA control; * $=\mathrm{p}<0.05$ ).
ChipSeq Encode data at the CTCFBSDB2.0 site revealed that CTCF binds the AATK CpG island in HUVEC. Furthermore, a search for potential CTCF-binding sites at the AATK locus found a match for a CTCF binding consensus site from position 79'139'590 to 79'139'599 (CCGCCAGGG) at $\mathrm{CpG}$ site 2 (Fig. 1A) (http:// bsproteomics.essex.ac.uk: 8080/bioinformatics/ctcfbind. $\mathrm{htm})$. To clarify whether CTCF could regulate AATK expression, we transfected CTCF in HeLa, A549 and H322 cancer cells (Fig. 4). Expression of CTCF induced the expression of AATK considerably in all three cell lines (Fig. 4). For H322 cells a 3.3 time increase in AATK expression was observed (Fig. 4). Since CTCF consist of three distinct domains: an N-terminal domain (NT), a zinc finger domain (ZF), which binds to DNA and a C-terminal (CT) domain. We generated deletion constructs of CTCF to investigate which of these domains is responsible for reactivation of AATK. Subsequently, we transfected these deletion constructs (CT-, NT- and ZF-CTCF), full length CTCF, and the empty EGFP vector control in H322 lung cancer cells (Fig. 5). Transfection of CTCF, NT-CTCF and ZF-CTCF induced the expression of AATK to levels similar to that of the control (Fig. 5A). Interestingly, a repression of AATK expression occurred for CT-CTCF transfection. To reveal if this induction is accompanied by changes in methylation levels we performed bisulfite pyrosequencing of three $\mathrm{CpG}$ sites (CpG1, $\mathrm{CpG} 2$ and $\mathrm{CpG} 3$ ) around the CTCF consensus site (Fig. 1 A and Fig. 5B). Interestingly, we observed a slight and significant decrease from $84 \%$ to $79 \%$ average methylation of the three CpGs after control and CTCF transfection, respectively ( $\mathrm{p}=0.03$, t-test; Fig. 5B). This decrease was higher for $\mathrm{CpG} 1$ ( $89 \%$ to $83 \%$; $\mathrm{p}=0.03)$ and $\mathrm{CpG} 2(79 \%$ to $71 \%, \mathrm{p}=0.3)$, than for the third CpG (Fig. 5B). Similar demethylation was also observed after CT-CTCF, NT-CTCF and ZF-CTCF transfection (Fig. 5B). It is interesting to note that demethylation of the CpG site 2, which harbors the CTCF consensus site, was more pronounced after CTCF and NT-CTCF transfection compared to CT-CTCF and ZF-CTCF. In summary, we observed a reproducible demethylation of AATK after CTCF overexpression.

\section{AATK expression reduces colony formation of cancer cells}

To functionally test AATK and its ability to suppress tumor formation, we performed colony formation and proliferation assays (Fig. 6). In order to do so we transfected HeLa and H322 with the Aatk expression- or empty control construct (pCDNA3.1) and selected with G418 for three weeks. Colonies were Giemsa stained and representative pictures are shown (Fig. 6A). In both cell lines $A A T K$ is methylated and downregulated (Fig. 1 and 3). After transfection of the Aatk containing construct, its expression was detected on RNA level (Fig. 6D). Expression of Aatk in these two cell lines significantly 
reduces the number of colonies (Fig. 6B). For both cell lines a twofold reduction in colonies was found (Fig. 6B). Moreover we analyzed proliferation of H322 cells after transient Aatk transfection. Cells were transfected with Aatk or empty vector (pCDNA3.1) and were counted over three days (Fig. 6D). After 24 hours a 25\% reduction in cell number was found, however this reduction was not significant due to the observed variation $(\mathrm{p}=0.4)$. The significant reduction of colonies suggests that Aatk exhibits a tumor suppressive function in cancer cells.

\section{DISCUSSION}

In our study, we have identified the apoptosis associated tyrosine kinase (AATK) gene as a novel epigenetically inactivated target gene in human cancer. Hypermethylation of $A A T K$ was found in several epithelial cancer entities including lung, breast, skin, cervix, larynx and pancreatic cancer (Fig. 1 and Fig. 2). However, expression of AATK was found in normal tissues and its $\mathrm{CpG}$ island was unmethylated (Fig. 2). We observed that

A

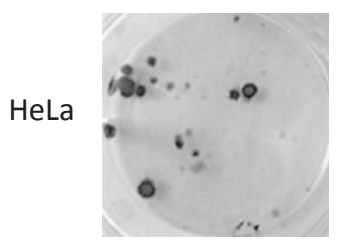

pCDNA3.1

H322

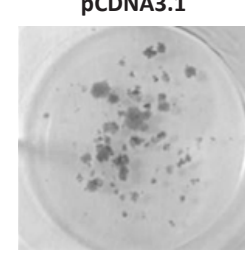

B
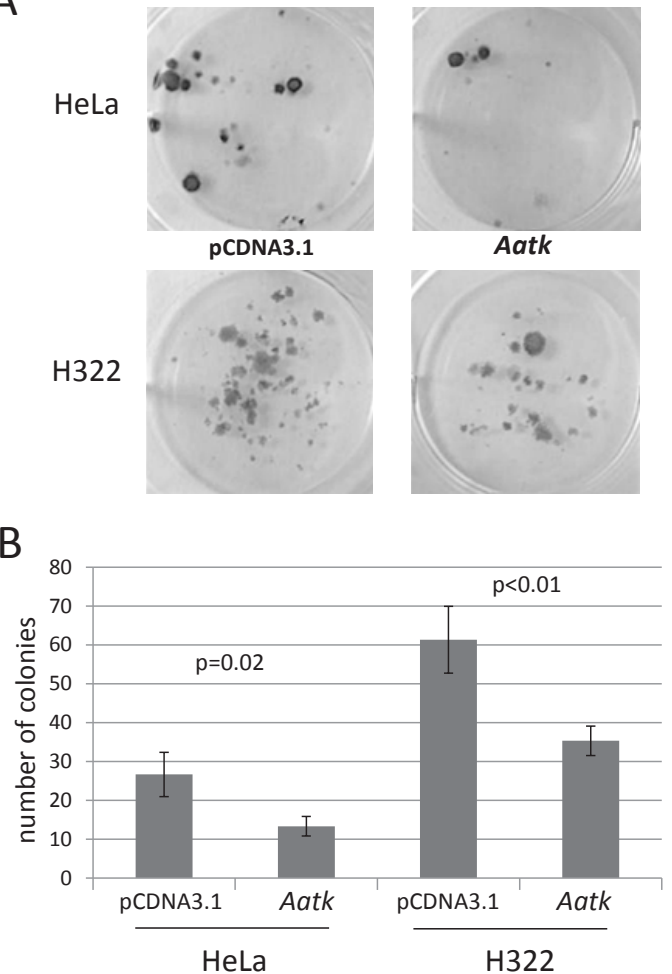

aberrant methylation of $A A T K$ is also frequently found in primary lung cancer (40\%) and breast cancers (53\%). Methylation frequency was significantly lower in matching normal tissues compared to breast cancers $(17 \%$ compared to $53 \%, \mathrm{p}<0.01)$. It is interesting to note that deletion of the chromosomal region which harbors the AATK gene is observed in different tumor entities including breast, cervix and oral cancers [14-16]. Since in general both alleles of a tumor suppressor gene are inactivated, these findings indicate that deletion and epigenetic inactivation are two important pathways for the inactivation of AATK in human cancer. It will be interesting to analyze if single nucleotide substitutions are present in the coding or regulatory sequences of $A A T K$. Previously, downregulation of AATK at the protein level was only observed in colon polyps and melanoma, however the mechanism responsible for this downregulation was not revealed in detail $[20,21]$. Thus, AATK hypermethylation may also be present in colon cancer and melanoma, as observed for the RASSF1A gene and other tumor suppressor genes [2527]. Methylation of $A A T K$ has been reported in low grade

C

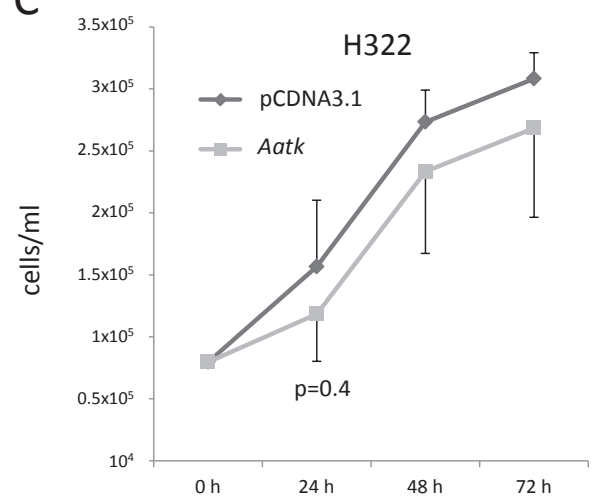

D

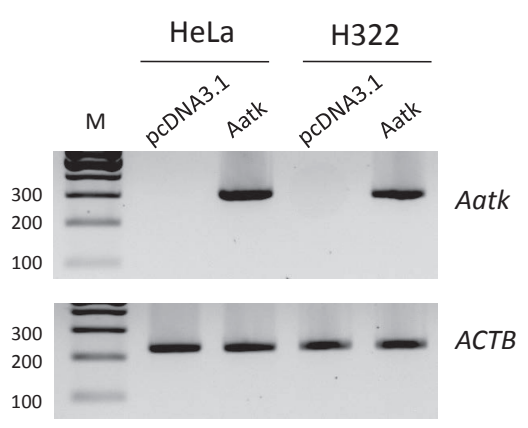

Fig. 6: Overexpression of Aatk reduces colony formation and proliferation of cancer cell lines. A. Colony formation after Aatk reexpression in cancer cells. HeLa and H322 lung cancer cell lines were transfected with Aatk or empty vector (pCDNA3.1), selected with G418 for 28 days and Giemsa stained. B. Colony formation experiment was repeated three times, and the mean colony numbers with according SD are shown for HeLa and H322 cells. P values were calculated using two tailed t-test. C. Proliferation of H322 cells after transient Aatk transfection. $0.8 \times 10^{5} \mathrm{cells} / \mathrm{ml}$ were transfected with Aatk or empty vector (pCDNA3.1) and cells were counted for three days in a hemocytometer. Experiments were performed in triplicate and the corresponding mean and SD are plotted. D. Overexpression of Aatk was confirmed by RT-PCR in HeLa and H322 cells. HeLa and H322 cells were transfected with the indicated constructs and RNA was isolated. Aatk (290 bp) and ACTB (226 bp) levels were analyzed on a $\%$ gel together with a 100 bp marker (M). 
serous ovarian neoplasms [28]. Here we report that $A A T K$ hypermethylation is frequently found in other human epithelial cancers, including primary breast and lung cancers (Fig. 2). Thus, it will be interesting to evaluate whether aberrant $A A T K$ methylation may represent a novel biomarker for prognostic or diagnostic purposes in human cancer.

By using 5-aza-2'-deoxycytidine treatment we were able to demethylate the $A A T K \mathrm{CpG}$ island promoter and to re-express $A A T K$ (Fig. 3). This suggests that downregulation of $A A T K$ in cancer cell lines is due to its promoter hypermethylation. Moreover, we observed that expression of the epigenetic regulator $\mathrm{CTCF}$ also induced the expression and demethylation of AATK (Fig. 4 and Fig. 5). Previously, it has been reported that knockdown of $C T C F$ by RNA interference caused twofold repression of $A A T K$ in HeLa cells $[23,24]$. Here we show that overexpression of CTCF induced the expression of $A A T K$ in HeLa and in the lung cancer cell lines A549 and H322. It has been postulated that CTCF acts as a tumor suppressive factor $[29,30]$, since its function has been associated with altered expression of tumor-suppressor gene, such as E-cadherin, retinoblastostoma, RASSF $1 A$ or $p 16 / C D K N 2 A$ $[10,12]$. Disrupting the spectrum of target specificities of CTCF by mutations, its aberrant modifications (e.g. PARylation) or abnormal selective methylation of targets (e.g. loss of imprinting) could be associated with cancer $[12,31,32]$. Recurrent mutations of CTCF are mostly clustered in the conserved zinc finger domain [30]. It has been reported that CTCF-defective PARylation and dissociation from the molecular chaperone Nucleolin occur in p16-silenced cells, abrogating its function [12]. Using CTCF mutants, the requirement of PARylation for optimal CTCF function has been demonstrated in transcriptional activation of the $p 19 A R F$ promoter and inhibition of cell proliferation [33]. In our experiments we observed that full length CTCF and the N-terminal domain of CTCF, which harbor a intact PARylation site, induce the expression of $A A T K$. In contrast, the $\mathrm{C}$-terminal site of CTCF repressed $A A T K$ expression. CTCF has also been reported to act as a gene silencer [34]. Thus, the function of $\mathrm{CTCF}$ may require a specific composition of factors (e.g. PARP1) at CTCF binding sites. This has been suggested in a model where PARylation of CTCF is involved in gene activation [12]. Others have reported that SUMOylation of CTCF modulates a domain in CTCF that activates transcription and decondenses chromatin [35]. One SUMOylation site is also found in the N-terminal domain of CTCF. Thus, CTCF may act as a tumor relevant factor by inducing tumor relevant genes such as $A A T K$. In the case of $A A T K$ this induction was also accompanied by a significant demethylation (Fig. 5B). Chipseq data reveal a CTCF binding site within the analyzed sequence. Thus, it will be interesting to analyze if increased CTCF binding occurs at its binding site upon CTCF expression.
It has been shown that CTCF bound DNA remains unmethylated [36]. Stadler et al. showed that CTCF can bind to a pre-methylated CpG-poor target site, which in turn leads to localized demethylation [36]. However the exact mechanism of this local demethylation event has not been elucidated, and it could be passive by inhibiting the accessibility of binding sites for DNA methyltransferases during DNA replication. For this aspect, it is important to note that the demethylation caused by CTCF (5\%) was half as much compared to Aza (10\%), however Aza treatment took twice as long (4 days) compared to CTCF transfection (2 days). Additional chromatin changes (e.g. histone modification or structural alteration) could be involved in the CTCF-induced expression of AATK and should to be analyzed in further studies.

To verify the ability of AATK to suppress tumor growth as do other tumor suppressor genes, we performed colony formation and proliferation assays in human cancer cell lines (Fig. 6). Our results show that Aatk significantly suppresses colony growth in H322 lung cancer cells and HeLa cells, in which AATK is downregulated and inactivated by aberrant promoter methylation (Fig. 1 and Fig. 2). Recently, it has been reported that AATK inhibits cell proliferation, colony formation, migration, and also promotes apoptosis in melanoma cells [21]. Since we observed hypermethylation of AATK in IGR1 melanoma cell lines (Fig. 1), it will be interesting to analyze its methylation status in primary melanomas.

AATK was characterized as a novel kinase that induces and promotes neuronal differentiation in a human neuroblastoma cell line [19]. Thus it will be important to analyze if downregulation of AATK is associated with dedifferentiation of human epithelial cells. It has been reported that AATK interacts with the p35 activation subunit of the cyclin-dependent kinase 5 (CDK5) and is then phosphorylated by CDK5 $[37,38]$. It has been suggested that AATK is a regulator of axonal outgrowth involving the RAB11 endosomal recycling pathway [17]. However, it is not known whether AATK phosphorylates any target proteins, and the function of AATK in epithelial cells has not been analyzed in detail.

In our study we demonstrate that $A A T K$ is frequently hypermethylated in human cancer cell lines and in primary lung and breast cancer samples. Demethylation of AATK is accompanied by re-expression of $A A T K$ in cancer cell lines. AATK expression was further found to be epigenetically regulated by CTCF. Additionally, ectopic expression of AATK suppresses colony formation. It will be fascinating to investigate the functional relationship of $A A T K$ epigenetic inactivation in cancer cell lines and their inability to differentiate or to undergo apoptosis. Therefore the loss of $A A T K$ might even promote dedifferentiation during tumorigenesis. Future research will elucidate the function of AATK during carcinogenesis. 


\section{MATERIALS AND METHODS}

\section{Tissue and cell lines}

Primary cancer tissues and cancer cell lines were previously published: breast and lung [39-42]. All patients signed informed consent at initial clinical investigation. The study was approved by local ethic committees (City of Hope Medical Center, Duarte, USA and Martin-Luther University, Halle, Germany). All cell lines were cultured in a humidified atmosphere $\left(37^{\circ} \mathrm{C}\right)$ with $5 \% \mathrm{CO}_{2}$ and $1 \mathrm{xPenicillin} / \mathrm{Streptomycin}$ in the recommended medium. Cells were transfected with $4 \mu \mathrm{g}$ or $10 \mu \mathrm{g}$ of constructs for 3.5 or $10 \mathrm{~cm}$ plates, respectively using Polyethylenimine or Turbofect (Fermentas GmbH, St.Leon-Rot, Germany).

\section{Methylation analysis}

DNA was isolated by phenol-chloroform extraction and then bisulfite treated prior to COBRA analysis and pyrosequencing [43]. $200 \mathrm{ng}$ were subsequently used for PCR with primer AATKBSU1 (GGTTTGTATGGAAATTAATTTTTTTTT)

and 5'-biotinylated primer AATKBSL2 (ATTTATACTAAAACCCAAAACCTACCC). Products were digested with $0.5 \mu \mathrm{l}$ TaqI (Fermentas $\mathrm{GmbH}$, St.Leon-Rot, Germany) $1 \mathrm{~h}$ at $65^{\circ} \mathrm{C}$ and resolved on $2 \%$ TBE gel. Methylation status was quantified utilizing the primer AATKSeq1 (GAGTTTAGTAGTAGAAGTAGT) and PyroMark Q24 (Qiagen, Hilden, Germany). Three CpGs are included in analyzed region of AATK and mean methylation was calculated. For in vitro methylation of genomic DNA we used M.SssI methylase (NEB, Frankfurt, Germany).

\section{Expression analysis}

RNA was isolated using the Isol-RNA lysis procedure (5 Prime, Hamburg, Germany). $25 \mu \mathrm{g}$ of breast, kidney, liver and lung RNA of normal human samples (pools of five, one, three and four, respectively) were obtained from Agilent Technologies (Waldbronn, Germany). RNA was DNase (Fermentas GmbH, St.LeonRot, Germany) digested and then reversely transcribed [44]. RT-PCR was performed with primers: AATKRTF1: TGGCCTGGCTCACTGCAAGTACAG, AATKRTR1: CCCAGATGGTCACGCCCAGG, mAatkRTF1: GTGCTGAAGTGACCCCCTAC, mAatkRTR1: GGTCAGCGGTCACGAGATAG, BACTFW: CCTTCCTTCCTGGGCATGGAGTC, BACTRW: CGGAGTACTTGCGCTCAGGAGGA, GGCTCFRTFW: CAGGAAACGGAGGCTACGGTGG, GGCTCFRTRW: CCTCCTGCAGGCCTCCTTTGGA. Quantitative PCR
(qRT-PCR) was performed in triplicate with PerfeCTa SYBR ${ }^{\circledR}$ Green (Quanta BioSciences, Gaithersburg, USA) using a Rotor-Gene 3000 (Corbett Research, Qiagen, Hilden, Germany).

\section{Constructs}

The cDNA of Aatk was obtained as a full length cDNA vector IRAVp968F06121D (Accession Number: BC080846; 5277 bp in pYX-Asc; imaGenes $\mathrm{GmbH}$, Berlin, Germany) and cloned into the NotI and EcoRI sites of pcDNA3.1+. CTCF was a generous gift from Rainer Renkawitz (Justus Liebig University, Giessen, Germany) and deletion mutations were generated with QuickChange Lightning Site-Directed Mutagenesis Kit (Promega, Heidelberg, Germany).

\section{List of Abbreviations}

AATK: apoptosis associated tyrosine kinase; COBRA: combined bisulfite restriction analysis; Aza: 5-aza-2'-deoxycytidine, CTCF: CCCTC binding factor

\section{Competing interests}

The authors declare that they have no competing interests. The work was supported by grants (TRR81, LOEWE) from the DFG and Land Hessen to Reinhard Dammann. These organizations had no involvement in the study design, acquisition, analyses, data interpretation, writing of the manuscript and in the decision to submit the manuscript for publication.

\section{Authors' contribution}

RHD has created the study. TH and RHD participated in the design of the study. TH, SKW, AMR and $\mathrm{CH}$ acquired data. TH, CH, SKW, AMR and RHD controlled, analyzed, and interpreted data. RHD prepared the manuscript. TH, CH, SKW, AMR and RHD read, corrected and approved the final manuscript.

\section{ACKNOWLEDGEMENTS}

The work was supported by grants (TRR81, LOEWE) from the DFG and Land Hessen to Reinhard Dammann. These organizations had no involvement in the study design, acquisition, analyses, data interpretation, writing of the manuscript and in the decision to submit the manuscript for publication. We would like to thank Rajkumar Savai for generous gift of materials and Helmut Dotzlaw for carefully reading the manuscript. 


\section{REFERENCES}

1. Jones PA and Baylin SB. The epigenomics of cancer. Cell. 2007; 128(4):683-692.

2. Dammann R, Li C, Yoon JH, Chin PL, Bates S and Pfeifer GP. Epigenetic inactivation of a RAS association domain family protein from the lung tumour suppressor locus 3p21.3. Nat Genet. 2000; 25(3):315-319.

3. Richter AM, Pfeifer GP and Dammann RH. The RASSF proteins in cancer; from epigenetic silencing to functional characterization. Biochim Biophys Acta. 2009; 1796(2):114-128.

4. Herold M, Bartkuhn M and Renkawitz R. CTCF: insights into insulator function during development. Development. 2012; 139(6):1045-1057.

5. Butcher DT, Mancini-DiNardo DN, Archer TK and Rodenhiser DI. DNA binding sites for putative methylation boundaries in the unmethylated region of the BRCA1 promoter. Int J Cancer. 2004; 111(5):669-678.

6. Fan S, Fang F, Zhang X and Zhang MQ. Putative zinc finger protein binding sites are over-represented in the boundaries of methylation-resistant $\mathrm{CpG}$ islands in the human genome. PLoS ONE. 2007; 2(11):e1184.

7. Filippova GN, Cheng MK, Moore JM, Truong JP, Hu YJ, Nguyen DK, Tsuchiya KD and Disteche CM. Boundaries between chromosomal domains of $\mathrm{X}$ inactivation and escape bind CTCF and lack CpG methylation during early development. Dev Cell. 2005; 8(1):31-42.

8. Ishihara $\mathrm{K}$, Oshimura $\mathrm{M}$ and Nakao M. CTCF-dependent chromatin insulator is linked to epigenetic remodeling. Mol Cell. 2006; 23(5):733-742.

9. Burke LJ, Zhang R, Bartkuhn M, Tiwari VK, Tavoosidana G, Kurukuti S, Weth C, Leers J, Galjart N, Ohlsson R and Renkawitz R. CTCF binding and higher order chromatin structure of the H19 locus are maintained in mitotic chromatin. Embo J. 2005; 24(18):3291-3300.

10. Davalos-Salas M, Furlan-Magaril M, Gonzalez-Buendia E, Valdes-Quezada C, Ayala-Ortega E and Recillas-Targa F. Gain of DNA methylation is enhanced in the absence of CTCF at the human retinoblastoma gene promoter. BMC cancer. 2011; 11:232.

11. De La Rosa-Velazquez IA, Rincon-Arano H, BenitezBribiesca L and Recillas-Targa F. Epigenetic regulation of the human retinoblastoma tumor suppressor gene promoter by CTCF. Cancer Res. 2007; 67(6):2577-2585.

12. Witcher $M$ and Emerson BM. Epigenetic silencing of the p16(INK4a) tumor suppressor is associated with loss of CTCF binding and a chromatin boundary. Mol Cell. 2009; 34(3):271-284.

13. Seki N, Hayashi A, Hattori A, Kozuma S, Ohira M, Hori $\mathrm{T}$ and Saito T. Chromosomal assignment of a human apoptosis-associated tyrosine kinase gene on chromosome $17 q 25.3$ by somatic hybrid analysis and fluorescence in situ hybridization. Journal of human genetics. 1999; 44(2):141142.

14. Saito H, Inazawa J, Saito S, Kasumi F, Koi S, Sagae S, Kudo R, Saito J, Noda K and Nakamura Y. Detailed deletion mapping of chromosome $17 \mathrm{q}$ in ovarian and breast cancers: 2-cM region on 17q21.3 often and commonly deleted in tumors. Cancer Res. 1993; 53(14):3382-3385.

15. Viswanathan M, Sangiliyandi G, Vinod SS, Mohanprasad BK and Shanmugam G. Genomic instability and tumorspecific alterations in oral squamous cell carcinomas assessed by inter-(simple sequence repeat) PCR. Clinical cancer research : an official journal of the American Association for Cancer Research. 2003; 9(3):1057-1062.

16. Yu J, Zhou H, Jin Y, Bai J, Yu Y, Geng J, Qi J and Fu S. Three distinct regions of allelic deletion on chromosome 17 involved in sporadic gastric cancer. Hepatogastroenterology. 2008; 55(85):1487-1491.

17. Takano T, Tomomura M, Yoshioka N, Tsutsumi K, Terasawa Y, Saito T, Kawano H, Kamiguchi H, Fukuda M and Hisanaga S. LMTK1/AATYK1 is a novel regulator of axonal outgrowth that acts via Rab11 in a Cdk5-dependent manner. The Journal of neuroscience : the official journal of the Society for Neuroscience. 2012; 32(19):6587-6599.

18. Gaozza E, Baker SJ, Vora RK and Reddy EP. AATYK: a novel tyrosine kinase induced during growth arrest and apoptosis of myeloid cells. Oncogene. 1997; 15(25):31273135.

19. Raghunath M, Patti R, Bannerman P, Lee CM, Baker S, Sutton LN, Phillips PC and Damodar Reddy C. A novel kinase, AATYK induces and promotes neuronal differentiation in a human neuroblastoma (SH-SY5Y) cell line. Brain research Molecular brain research. 2000; 77(2):151-162.

20. Lee S, Bang S, Song K and Lee I. Differential expression in normal-adenoma-carcinoma sequence suggests complex molecular carcinogenesis in colon. Oncology reports. 2006; 16(4):747-754.

21. Ma S and Rubin BP. Apoptosis-associated tyrosine kinase 1 inhibits growth and migration and promotes apoptosis in melanoma. Laboratory investigation; a journal of technical methods and pathology. 2014; 94(4):430-438.

22. Jones PA and Taylor SM. Cellular differentiation, cytidine analogs and DNA methylation. Cell. 1980; 20(1):85-93.

23. Barski A, Cuddapah S, Cui K, Roh TY, Schones DE, Wang Z, Wei G, Chepelev I and Zhao K. High-resolution profiling of histone methylations in the human genome. Cell. 2007; 129(4):823-837.

24. Wendt KS, Yoshida K, Itoh $\mathrm{T}$, Bando $\mathrm{M}$, Koch B, Schirghuber E, Tsutsumi S, Nagae G, Ishihara K, Mishiro T, Yahata K, Imamoto F, Aburatani H, Nakao M, Imamoto $\mathrm{N}$, Maeshima K, et al. Cohesin mediates transcriptional insulation by CCCTC-binding factor. Nature. 2008; 451(7180):796-801. 
25. Yoon JH, Dammann R and Pfeifer GP. Hypermethylation of the $\mathrm{CpG}$ island of the RASSF1A gene in ovarian and renal cell carcinomas. Int J Cancer. 2001; 94(2):212-217.

26. Rastetter M, Schagdarsurengin U, Lahtz C, Fiedler E, Marsch W, Dammann R and Helmbold P. Frequent intratumoural heterogeneity of promoter hypermethylation in malignant melanoma. Histol Histopathol. 2007; 22(9):10051015.

27. Helmbold P, Richter AM, Walesch S, Skorokhod A, Marsch W, Enk A and Dammann RH. RASSF10 promoter hypermethylation is frequent in malignant melanoma of the skin but uncommon in nevus cell nevi. J Invest Dermatol. 2012; 132(3 Pt 1):687-694.

28. Shih Ie M, Chen L, Wang CC, Gu J, Davidson B, Cope L, Kurman RJ, Xuan J and Wang TL. Distinct DNA methylation profiles in ovarian serous neoplasms and their implications in ovarian carcinogenesis. American journal of obstetrics and gynecology. 2010; 203(6):584 e581-522.

29. Fiorentino FP and Giordano A. The tumor suppressor role of CTCF. Journal of cellular physiology. 2012; 227(2):479492.

30. Tiffen JC, Bailey CG, Marshall AD, Metierre C, Feng Y, Wang Q, Watson SL, Holst J and Rasko JE. The cancertestis antigen BORIS phenocopies the tumor suppressor CTCF in normal and neoplastic cells. Int J Cancer. 2013; 133(7):1603-1613.

31. Dammann RH, Kirsch S, Schagdarsurengin U, Dansranjavin T, Gradhand E, Schmitt WD and Hauptmann S. Frequent aberrant methylation of the imprinted IGF2/H19 locus and LINE1 hypomethylation in ovarian carcinoma. International journal of oncology. 2010; 36(1):171-179.

32. Ohlsson R, Renkawitz R and Lobanenkov V. CTCF is a uniquely versatile transcription regulator linked to epigenetics and disease. Trends in genetics : TIG. 2001; 17(9):520-527.

33. Farrar D, Rai S, Chernukhin I, Jagodic M, Ito Y, Yammine S, Ohlsson R, Murrell A and Klenova E. Mutational analysis of the poly(ADP-ribosyl)ation sites of the transcription factor CTCF provides an insight into the mechanism of its regulation by poly(ADP-ribosyl)ation. Mol Cell Biol. 2010; 30(5):1199-1216

34. Arnold R, Maueler W, Bassili G, Lutz M, Burke L, Epplen TJ and Renkawitz R. The insulator protein CTCF represses transcription on binding to the $(\mathrm{gt})(22)(\mathrm{ga})(15)$ microsatellite in intron 2 of the HLA-DRB1(*)0401 gene. Gene. 2000; 253(2):209-214.

35. Kitchen NS and Schoenherr CJ. Sumoylation modulates a domain in CTCF that activates transcription and decondenses chromatin. Journal of cellular biochemistry. 2010; 111(3):665-675.

36. Stadler MB, Murr R, Burger L, Ivanek R, Lienert F, Scholer A, van Nimwegen E, Wirbelauer C, Oakeley EJ, Gaidatzis D, Tiwari VK and Schubeler D. DNA-binding factors shape the mouse methylome at distal regulatory regions. Nature. 2011; 480(7378):490-495.

37. Honma N, Asada A, Takeshita S, Enomoto M, Yamakawa E, Tsutsumi K, Saito T, Satoh T, Itoh H, Kaziro Y, Kishimoto $\mathrm{T}$ and Hisanaga $\mathrm{S}$. Apoptosis-associated tyrosine kinase is a Cdk5 activator p35 binding protein. Biochem Biophys Res Commun. 2003; 310(2):398-404.

38. Tsutsumi K, Takano T, Endo R, Fukuda M, Ohshima T, Tomomura $\mathrm{M}$ and Hisanaga S. Phosphorylation of AATYK1 by Cdk5 suppresses its tyrosine phosphorylation. PLoS One. 2010; 5(4):e10260.

39. Schagdarsurengin U, Pfeifer GP and Dammann R. Frequent epigenetic inactivation of cystatin $\mathrm{M}$ in breast carcinoma. Oncogene. 2007; 26(21):3089-3094.

40. Dammann R, Strunnikova M, Schagdarsurengin U, Rastetter M, Papritz M, Hattenhorst UE, Hofmann HS, Silber RE, Burdach S and Hansen G. CpG island methylation and expression of tumour-associated genes in lung carcinoma. Eur J Cancer. 2005; 41(8):1223-1236.

41. Dammann R, Yang G and Pfeifer GP. Hypermethylation of the cpG island of Ras association domain family $1 \mathrm{~A}$ (RASSF1A), a putative tumor suppressor gene from the 3 p21.3 locus, occurs in a large percentage of human breast cancers. Cancer Res. 2001; 61(7):3105-3109.

42. Helmbold P, Lahtz C, Herpel E, Schnabel PA and Dammann RH. Frequent hypermethylation of RASSF1A tumour suppressor gene promoter and presence of Merkel cell polyomavirus in small cell lung cancer. Eur J Cancer. 2009; 45(12):2207-2211.

43. Dammann G, Teschler S, Haag T, Altmuller F, Tuczek $\mathrm{F}$ and Dammann RH. Increased DNA methylation of neuropsychiatric genes occurs in borderline personality disorder. Epigenetics : official journal of the DNA Methylation Society. 2011; 6(12):1454-1462.

44. Richter AM, Walesch SK, Wurl P, Taubert H and Dammann RH. The tumor suppressor RASSF10 is upregulated upon contact inhibition and frequently epigenetically silenced in cancer. Oncogenesis. 2012; 1:e18. 